\title{
Conf- $910968--26$
}

UCRL- JC - 108485

PREPRINT

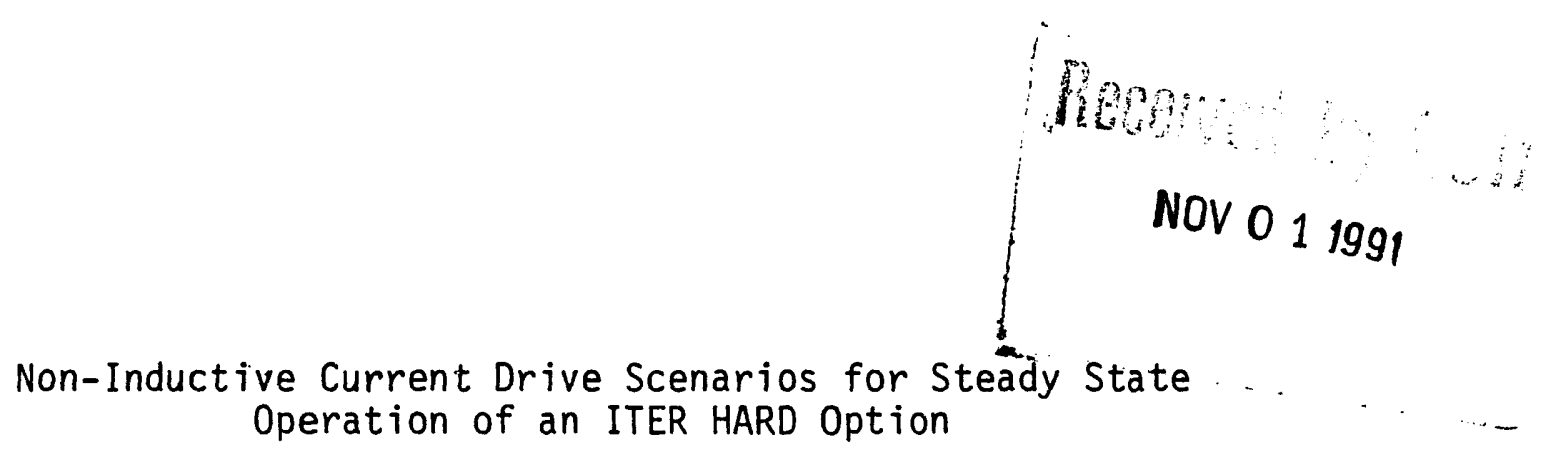

M. Fenstermacher, W. Nevins, R. Bulmer, W. Lindquist,

J. Parker, G. Smith,

W. Cooper, * R. Wells,*

D. Ehst**

This paper was prepared for submittal to

The 14th Symposium on Fusion Engineering

San Diego, CA

September 30 through October 3, 1991

September 25, 1991

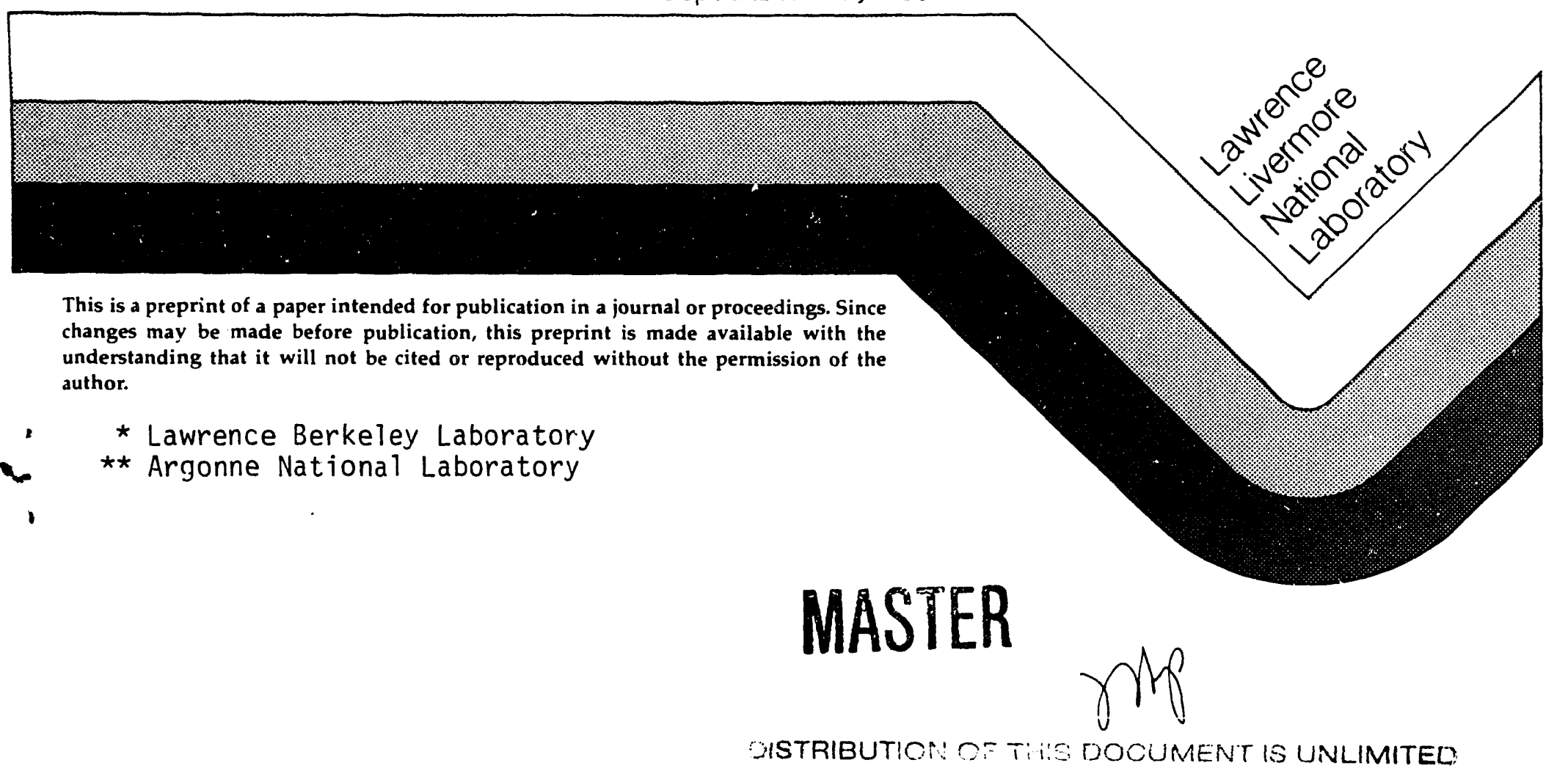




\section{DISCIAIMER}

This document was prepared as an account of work sponsored by an agency of the United States Government. Neither the United States Government nor the University of California nor any of their employees, makes any warranty; express or implied, or assumes any lepal liability or responsibility for the accuracy, completeness, or usefulmess of any information, apparatus, product, or process disclosed. or represents that its use would not infringe privately owned rights. Reference herein to any specific commercial products, process, or service by trade name. trademark, manufacturer, or otherwise, does not necessarily constitute or imply its endorsement, recommendation, or favoring by the United States Government or the University of California. The views and opinions of authors expressed herein do not necessarily state or reflect those of the United States Government or the University of California, and shall not be used for advertising or product endorsement purposes. 


\section{NON-INDUCTIVE CURRENT DRIVE SCENARIOS FOR STEADY STATE OPERATION OF AN ITER HARD OPTION*}

M. Fenstermacher, W. Nevins, R. Bulmer, W. Lindquist, J. Parker, G. Smith, Lawrence Livermore National Laboratory, Livermore, CA, 94551

W. Cooper and R. Wells, Lawrence Berkeley Laboratory, Berkeley, CA 94720

D. Ehst, Argonne National Laboratory, Argonne IL 60439.
UCRL-JC--108485

DE92 002165

\section{Abstract}

The requirements for steady state operation of a High Aspect Ratio Design (HARD) option for ITER using noninductive current driven by Neutral Beam (NB) injection, Lower Hybrid (LH) slow waves, and Fast Waves (FW) have been examined. For the higher field $\left(B_{T}=7.1 \mathrm{~T}\right)$ and moderate density $\left(\left\langle n_{e}\right\rangle=1.1 \times 10^{20} \mathrm{~m}^{-3}\right)$ in this HARD steady state operating point, NB injection provides the optimal current drive subject to the constraints of minimizing the total input power and achieving a high plasma internal inductance $\left(l_{i}=0.75\right.$; ie. a peaked current drive profile). An alternate scenario uses $F W$ current drive in the plasma core combined with LH waves in the outer plasma regions in place of the neutral beams, with approximately $30 \%$ higher power requirement. Finally, Electron Cyclotron (EC) resonance current drive is used in either scenario to provide localized perturbation of the current profile at the $q=2$ surface for disruption stabilization.

\section{Introduction}

In an attempt to improve the steady state performance of the ITER CDA design, the US Home Team has examined a HARD option for ITER $[1,2]$. The key differences between the HARD operating point chosen for this study and the CDA, which affect the current drive and heating (CD\&H) scenarios, are given in Table I. The most important of these are the higher density and lower temperature of the plasma which combine to make penetration of neutral beams to the plasma core more difficult and to reduce the current drive efficiency of all of the non-inductive current drive systems.

TABLE I. Key Differences Between CDA and HARD

\section{Parameter}

Major radius, $\mathrm{R}_{0}(\mathrm{~m})$

Minor radius, a (m)

Aspect ratio

Magnetic field, $B_{0}(T)$

Plasma current, $I_{p}$ (MA)

Average density, $<n_{e}>\left(10^{20} \mathrm{~m}^{-3}\right)$

Average temperature, $\left\langle\mathrm{T}_{\mathrm{e}}\right\rangle(\mathrm{keV})$

Internal inductance, $\mathrm{l}_{\mathrm{i} 3}$

Non-inductive current drive simulations for HARD have been performed within the context of two main goals:

1) The CD\&H system should drive current and create a

- Work performed under the auspices of the U.S. D.O.E. by LLNL under contract number W-7405-ENG-48. current profile with the reference value of the internal inductance, $\mathrm{l}_{\mathrm{i} 3}=0.75$, at a total plasma current of 11.1 MA.

2) The CD\&H system should allow variations in the driven current profile (at a fixed plasma current of 11.1 MA) to explore operation over a range of $l_{i 3}$ from the nominal value, $l_{13}=0.75$, down to the minimum allowed by the PF system design, $\mathrm{l}_{\mathrm{i} 3}=0.65$.

Note that for HARD, the stringent goal of achieving the nominal value of internal inductance was used; for the CDA the goal was only to achieve at least the minimum acceptable $\mathrm{l}_{\mathrm{i} 3}$. In addition to these physics goals, the general design philosophy in HARD was to minimize the required changes in the CD\&H systems developed for the ITER CDA (eg. NB energy fixed at $1.3 \mathrm{MeV}, \mathrm{FW}$ and LH systems essentially the same as in the CDA, etc.).

Numerical simulations of NB and LH current drive have been performed with the ACCOME code $[3,4]$. This program calculates self-consistent equilibria and driven current profiles by iterating between a current drive package and an equilibrium code. Calculations for scenarios using a combination of FW and LH current drive were done by iterating between a ray tracing package and an equlibrium solver.

In both of these calculations the Hirshman-Sigmar (H-S) bootstrap formalism [5] has been used for the HARD studies. This is in contrast to the calculations for the CDA in which the Hinton-Hazeltine $(\mathrm{H}-\mathrm{H})$ high aspect ratio formalism [6] was used. The H-S model significantly improves the bootstrap calculation by eliminating the assumption that $\left(a / R_{0}\right)^{1 / 2}$ is a small parameter. This assumption produces significant errors for both ITER and HARD throughout the plasma except near the axis. The H-S formalism also correctly treats multi-ion species contributions to the bootstrap current.

For a given equilibrium the Hirshman-Sigmar formalism gives a significantly higher fraction of bootstrap current (about $50 \%$ in the HARD design, up from about $30 \%$ in the CDA). This is a mixed blessing as it reduces the current that must be driven non-inductively (which is good), but also broadens the current profile-thus tending to reduce the internal inductance (which is a problem).

\section{Absence of Lower Hybrid System in the HARD Reference Scenario}

The CD\&H reference scenario of the ITER CDA design included a lower hybrid system. This system had two missions; driving current in the outer regions of the plasma to provide current profile control in steady-state or hybrid operation, and current ramp-up assist to extend the 
maximum achievable current for ignited operation to $I_{p}=$ $28 \mathrm{MA}$ (with $\mathrm{q}_{95}=2.34$ ) while maintaining a 200 second current flattop.

In both the CDA derign work and in our HARD study it was found that lower hybrid current drive is restricted to roughly the outer $1 / 3$ of the plasma ( $r / a>0.65$ for HARD). This is due to a combination of accessibility, which restricts the choice of refractive index $N_{1}$, and electron Landau damping, which determines a maximum $T_{e}$ that can be reached at at given $N_{1}$ (see Fig. 1).

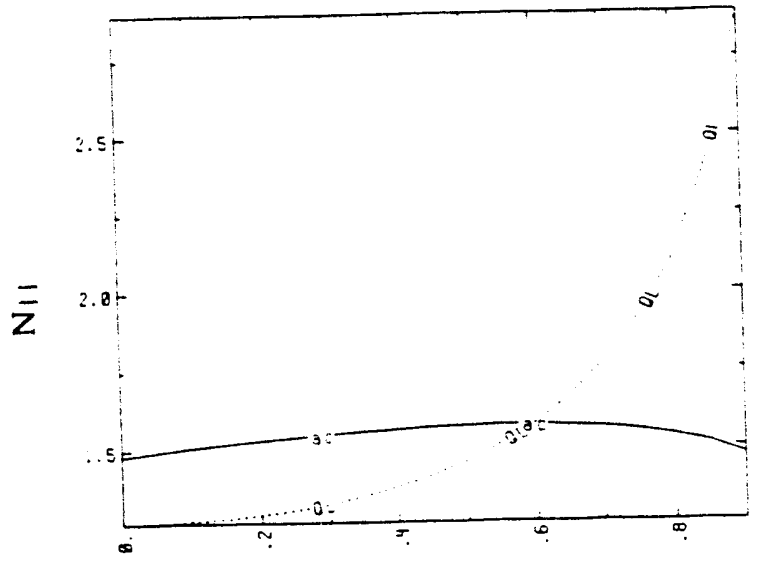

Normalized radius, r/a

Fig. 1 Wave propagation constraints for $\mathrm{LH}$ waves with parallel refractive index $N||$ as functions of normalized minor radius $\mathrm{r} / \mathrm{a} ; \mathrm{N}_{11, \mathrm{ac}}<\mathrm{N}_{11}<\mathrm{N}_{11, \mathrm{QL}}$ is required for propagation.

With the higher bootstrap fraction in HARD (about $50 \%$ as compared to about $30 \%$ in CDA) and the higher minimum target value of $l_{i 3}$ ( 0.65 for HARD as opposed to 0.55 for CDA design) there is less utility in driving current in the outer $1 / 3$ of the plasma-the neoclassical bootstrap effect already provides adequate current in this region. Detailed studies of combined LH and neutral beam (NB) current drive for a precurssor of the present HARD design $(R=6 \mathrm{~m}, A=4)$ indicated that significant amounts of $\mathrm{LH}$ power where only useful in steady-state scenarios with $l_{i 3}$ below 0.65 .

The HARD design provides a much greater margin in Volt-seconds than the CDA design. The plasma current can be ramped up inductively to $\mathrm{q}_{95}=2.34$ while still retaining 50 Volt-seconds for current flat-top. This higher current should provide a similar improvement in the HARD confinement to that achieved in the extended ignited operating scenario of the ITER CDA; while 50 Volt-seconds will allow $\sim 500$ seconds of current flat-top. Hence, a lower hybrid system is not required for current ramp-up assist in the HARD design.

Since the two motivations for a lower hybrid system in the CDA design (current profile control and non-inductive current ramp-up assist) are no longer present for HARD, the reference current drive and heating scenario does not include a lower hybrid system.

\section{Reference Steady-State Scenario}

An iterative process, involving ACCOME calculations and engineering design work [7], was used to develop the reference steady state CD\&H scenario for HARD. As described in Ref [7], the design uses 3 neutral beams/port with two stacked vertically and the third nested horizontally on the midplane between them. Various considerations that went into the process are described below.

\section{Aiming of the Neutral Beams}

The aiming of the neutral beams can be specified by

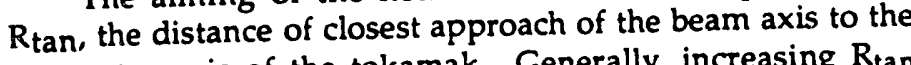
symmetry axis of the tokamak. Generally, increasing $R_{t a n}$ improves the neutral beam current drive figure-of-merit, $\gamma_{n b}$. Decreasing $R_{\tan }$ (at fixed beam energy) improves the penetration of the beam into the plasma by reducing the path length (and, hence, the line density) along the NB axis from the plasma edge to the magnetic axis of the tokamak.

An important trade-off occurs as $\mathbf{R}_{\tan }$ is varied. Lower values of $R_{\tan }$ give better beam penetration, a higher internal inductance, and a driven current profile that is less sensitive to changes in the plasma density. Larger values of $R_{\tan }$ give higher current drive efficiency, lower internal induciance, and larger bootstrap current fractions (which follow from the lower internal inductance).

In the ITER CDA design the neutral beam power requirements were minimized by choosing the largest value of $R_{\tan }$ consistent with central current drive at any density, $R_{\tan } / R_{0}-1$. At the relatively low density of the ITER CDA steady-state scenario $\left.\left(<n_{e}\right\rangle=0.64 \times 10^{20} \mathrm{~m}^{-3}\right)$ beam penetration was adequate at an energy of $1.3 \mathrm{MeV}$.

The HARD steady-state scenario has a substantially higher density $\left.\left(\left\langle n_{e}\right\rangle=1.1 \times 10^{20} m^{-3}\right)\right)$, so that neutral beam penetration becomes an issue. Beam penetration into the HARD plasma could be achieved by either increasing the beam energy, or by choosing a smaller value for the ratio $R_{\tan } / R_{O}$. To minimize the required changes to the CDA NB design, the same neutral beam energy was used for HARD as for the ITER CDA. Solutions, therefore, were examined with $R_{\tan } / R_{0}<1 . R_{\tan }=5.0 \mathrm{~m}$ was taken as a minimum acceptable value of the tangency radius. Aiming at a smaller radius would not ensure adequate clearance between the beam and the center post of the tokamak. This could lead to the deposition of substantial amounts of neutral beam power on the center post in low density operation. Aiming a significant fraction of the neutral beam power at a tangency radius of $5.0 \mathrm{~m}$ achieved an internal inductance of $\mathrm{l}_{\mathrm{i} 3}=0.74$.

\section{Beamline Length and Beam Footprint}

The length of the beamline and the aiming of individual beamlets in the source can have an impact on the current drive performance of the system. For HARD the high internal inductance requirement drove these decisions toward maximizing the current drive on the plasma axis. The two beams aimed at $R_{\tan }=5.0 \mathrm{~m}$ were given the minimum beamline length $(46.5 \mathrm{~m})$ needed to reduce the neutron flux at the sources to acceptable levels as 
determined during the CDA. Minimizing the beamline length reduces the beam divergence, increases the central peaking of the driven current, and reduces the beam scrapeoff on the tokamak center post for these beams. The third beam was aimed at $R_{\tan }=5.96 \mathrm{~m}$ with a beamline length of $52.2 \mathrm{~m}$. This value was determined from the geometry of the beam source structure [7]. The footprint projected to the tangency point without plasma was modelled in the ACCOME simulations by a Gaussian with $1 / \mathrm{e}$ half widths in the horizontal and vertical directions of $0.25 \mathrm{~m}$ and $0.60 \mathrm{~m}$, respectively.

\section{Current Drive Simulation Results}

Results from the ACCOME simulations of the reference $C D$ scenario are shown in Figs. 2-5. The fractional deposition profiles of the particles from the 3 beamlines are shown in Fig. 2 as functions of poloidal flux, $\psi$. Note that the profiles are similar for all of the beams. This is not unexpected since the beam path lengths in the plasma for the beams in the vertical stack are longer than for the single beam on the equitorial midplane.

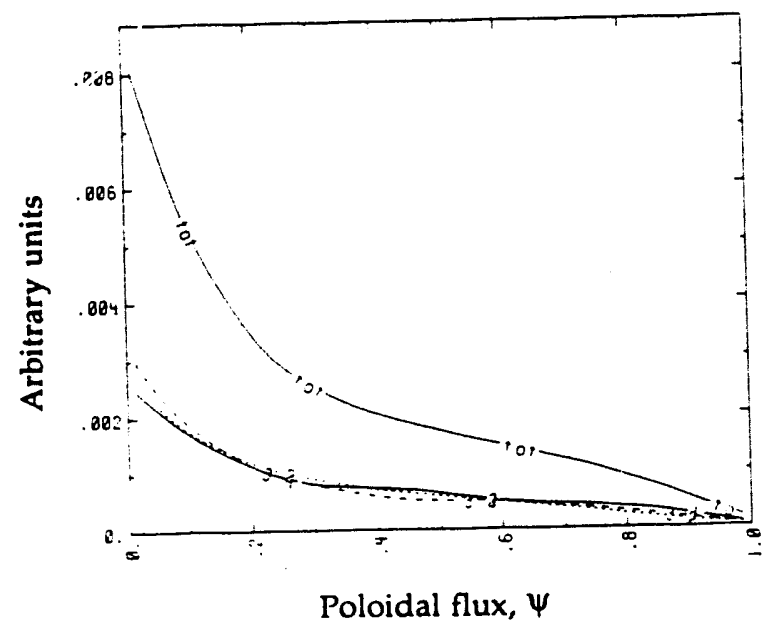

Fig. 2 Fractional particle deposition profiles vs. $\psi$ for beams with $R_{\tan }=5.96 \mathrm{~m}(1)$ and $R_{\tan }=5.0 \mathrm{~m}(2,3)$.

The components which sum to give the total plasma current density are shown in Fig. 3. The bootstrap effect provides the majority of the current in the outer half of the plasma and the beams provide the current near the plasma center. The beam driven current is the fast ion current minus the electron cancelling current. Flux surfaces and the contours of constant current density are shown in Figs. $4 \mathrm{a}$ and $4 \mathrm{~b}$, respectively.

The safety factor, $\psi$, as a function of $\psi$ is shown in Fig. 5. Note that the central value is less than 1.0 for $0<\psi<$ 0.2 so that sawteeth would be expected in this region. This modest sawtooth inversion radius should not greatly affect confinement. The low value of $q$ on axis results directly from the high internal inductance requirement in HARD.

\section{Electron Cyclotron Disruption Control}

The utility of electron cyclotron power for controlling disruptions by suppressing tearing modes at the $q=2$

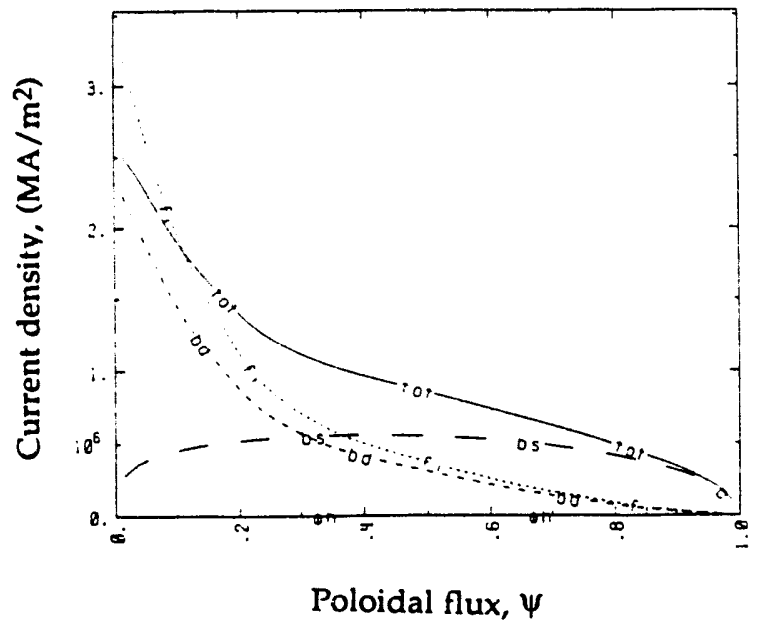

Fig. 3 Components of driven current density vs. $\psi$; beam driven (bd), bootstrap (bs), fast ion (fi) and total (tot). (a)

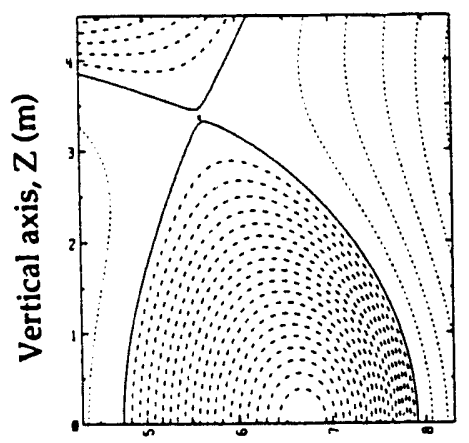

(b)

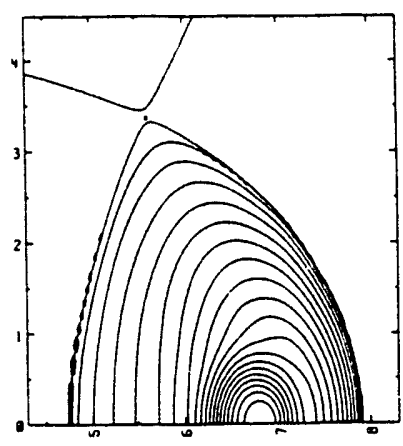

Major radius, $\mathrm{R}(\mathrm{m})$
Fig. 4 Flux surfaces (a) and current density contours (b) in the upper half plane $(R, Z)$ for the reference scenario.

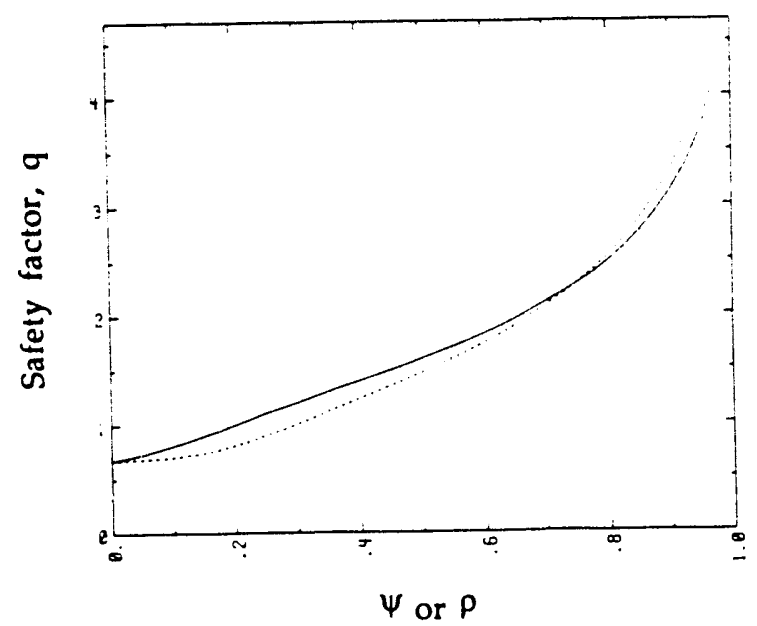

Fig. 5 Safety factor profile vs. $\psi$ (solid) and vs. toroidal flux $p$ (dashed). 
surface was studied for the HARD design. Earlier work on the CDA design had found EC power to be ineffective for this purpose at values of the plasma current exceeding 22 MA; it was also thought that the system might lack robustness for a range of currents below $22 \mathrm{MA}$. These problems are absent in the HARD design because the higher aspect ratio reduces trapped-electron degradation of current drive efficiency. Consequently the physics requirements for HARD were determined more completely than for the CDA.

The higher magnetic field of the HARD design leads to a higher frequency for the EC power. A frequency of 175 $\mathrm{GHz}$ was found to give optimal performance. The location of the driven current profile is dependent on both the frequency $f$, and the toroidal injection angle $\phi$, which is measured between the beam centerline and a radial drawn from the machine center at the entrance of the beam into the plasma.

Studies showed that EC power could suppress tearing modes on $q=2$ surfaces located at values of the normalized poloidal flux in the range $0.5<\psi<0.8$. This range could be covered by varying either the injection angle over the range $17^{\circ}<\mathrm{f}<23^{\circ}$ at fixed $\mathrm{f}=175 \mathrm{GHz}$ or by varying $\mathrm{f}$ over three to five values from 173 to $177 \mathrm{GHz}$ at fixed $\phi=20^{\circ}$.

Power requirements for tearing mode suppression are sensitive to the divergence of the beam. The divergence is most critical in the direction parallel to the magnetic field direction on the $q=2$ surface. In the equilibrium that was analyzed, this direction was inclined about $15^{\circ}$ with respect to the equatorial plane, implying that a vertical divergence has a parallel component. The power requirement is less than $25 \mathrm{MW}$ if the parallel divergence does not exceed $0.5^{\circ}$ but increases rapidly for larger divergence.

We also considered the effect of scattering of the EC power by drift-wave density fluctuations. This effect does not increase the power requirement for suppression of tearing modes because the scattering does not change the parallel wave-vector components of the EC waves.

\section{Alternate Current Drive and Heating Scenario}

Fast waves are effective for central current drive at high density, while central current drive with neutral beams becomes more difficult at the higher densities of the HARD steady state scenario. Hence, an alternate scenario using FW current drive in the plasma core would be expected to be relatively more attractive in the HARD design than in the CDA design.

Detailed modeling of steady state scenarios with a combination of lower hybrid driven, fast wave driven, and neoclassical bootstrap currents for the HARD design point were performed. Generally, it was found that the total current in steady state was determined by the lower hybrid power, while the addition of fast wave power led to an increase in the internal inductance at nearly constant plasma current. This behavior can be understood as a tradeoff between fast wave driven currents in the plasma core and the neoclassical bootstrap current. As the fast wave power is increased, the current density in the plasma core increases. This higher central current density has the effect of reducing the bootstrap current, so there is a corresponding reduction in the bootstrap current in the periphery of the plasma.

While this provides a convenient method of controlling the internal inductance, these calculations also indicate that this alternate scenario will require substantially more injected power than the reference scenario. The scenario employing neutral beams for bulk current drive retains a substantial advantage both in the required injected power and for current profile control. In the reference scenario $110 \mathrm{MW}$ of injected power was required for steady state at an internal inductance $l_{13}=0.74$, while about $140 \mathrm{MW}$ was required in the alternate scenario.

These studies motivate an alternate scenario with $35 \mathrm{MW}$ of lower hybrid power, and about $105 \mathrm{MW}$ of fast wave power. The resulting current drive and heating system is similar to that required for the ITER CDA. The lower hybrid system would require two half ports. The fast wave system could share the two lower hybrid ports, and would require an additional 5 ports. It is possible that, as in the CDA design, the fast wave system could share one or more of these ports with diagnostics and/or remote handling equipment. Given the increased power and port requirements for this alternate scenario, the neutral beam current drive scenario was chosen as the reference current drive and heating scenario for this HARD design.

\section{Conclusions}

A reference CD\&H scenario for steady state operation of a HARD option for ITER has been developed. It calls for $110 \mathrm{MW}$ of NB injection power at an energy of $E_{n b}=1.3$ $\mathrm{MeV}$, with two of the beams aimed at a tangency radius $R_{\tan }=5.0 \mathrm{~m}$ and a third aimed at $R_{\tan }=5.96 \mathrm{~m}$ in each of four NB ports. An alternate scenario employing $105 \mathrm{MW}$ of FW power, $35 \mathrm{MW}$ of $\mathrm{LH}$ power and using at least half of seven ports was found to produce similar steady state current drive performance. In either scenario, an EC system delivering about $25 \mathrm{MW}$ of power is used for local perturbation of the current profile at the $q=2$ surface to stabilize disruptions.

\section{REFERENCES}

1. L.J. Perkins, J.D. Galambos, et. al, "The International Experimental Reactor (ITER) at High Aspect Ratio: Is It Time to Consider a Change for the Engineering Design Activity?", LLNL Report UCRL-ID-104178, May 1990.

2. L.J. Perkins, et. al., Proc. $14^{\text {th }}$ IEEE Symp. on Fus. Eng., San Diego, 1991.

3. K. Tani, M. Azumi and R.S. Devoto, J. Comput. Phys. (1991) [to be published]

4. R.S. Devoto, D.T. Blackfield, M.E. Fenstermacher et. al., Nuc. Fusion., 1992 [to be published].

5. S. P. Hirshman and D.J. Sigmar, Nucl. Fusion 21, 1079 (1981).

6. F.L. Hinton and R.D. Hazeltine, Rev. Mod. Phys., 48, 239 (1976).

7. W. Lindquist, et. al., Proc. 14th IEEE Symp. on Fus. Eng., San Diego, 1991. 

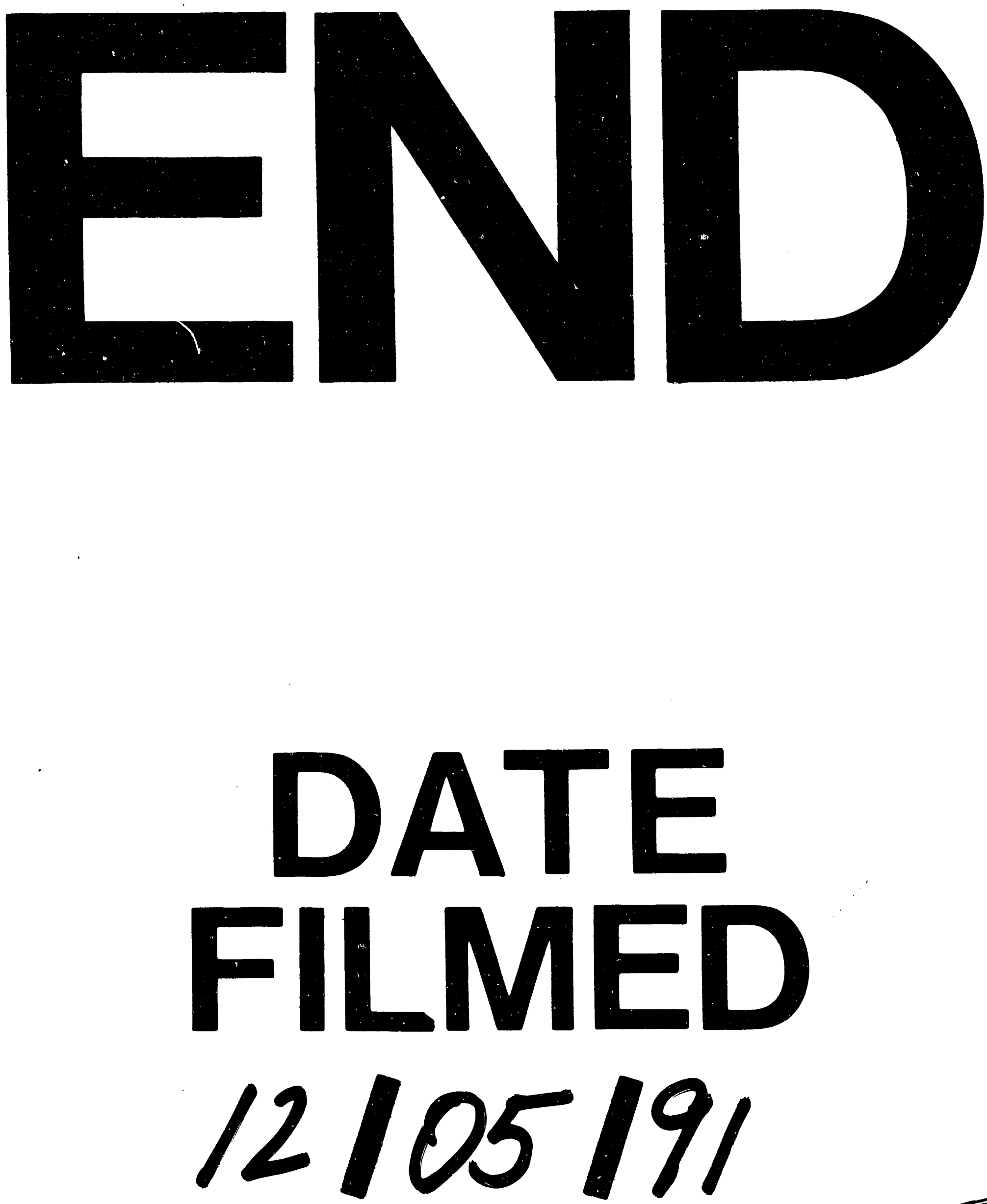

I 
